\title{
Educación Socioemocional: Descripción y Evaluación de un Programa de Capacitación de Profesores
}

\section{Socioemotional Education: Description and Evaluation of a Training Program for Teachers}

\author{
Teresita Marchant Orrego ${ }^{1}$ * \\ Neva Milicic Müller ${ }^{2}$ \\ Paulina Soto Vásquez ${ }^{3}$ \\ ${ }^{1}$ Fundación Belén Educa, Chile \\ ${ }^{2}$ Pontificia Universidad Católica de Chile, Chile \\ ${ }^{3}$ Universidad San Sebastián, Chile
}

\begin{abstract}
El objetivo de este artículo es describir un programa de desarrollo profesional docente, en el área de educación socioemocional y entregar algunos resultados de su impacto en la autoestima de los alumnos. Este se llevó a cabo en 12 colegios Particulares Subvencionados que atienden alumnos vulnerables económicamente, de Pre-Kínder (4 años) a Cuarto Medio (17 años), y que forman parte de una Fundación Educacional en Santiago, Chile. Este se desarrolló durante los años 2007-2017, en ciclos de dos años por colegio Los temas que se abordaron en la capacitación dada por expertos, con sesiones teóricas, talleres y materiales educativos, fueron definidos, con los respectivos colegios, como los más fundamentales a trabajar. Por ejemplo: Aprendizaje socioemocional en el contexto escolar; Variables que favorecen la construcción de identidad y autoestima positiva; Aporte de la neurodiversidad al desarrollo socioemocional; Resiliencia y aprendizaje socioemocional; Clima escolar y convivencia social; Intimidación escolar y desarrollo socioemocional; Aprendizaje socioemocional y bienestar docente. Se describe la implementación de este programa poniendo énfasis en el valor del aprendizaje socioemocional, en cómo desarrollar competencias en esta área y en cómo insertarlo en el contexto escolar. Se entregan los resultados de 2.865 alumnos evaluados pre-post intervención.
\end{abstract}

Palabras clave: Educación socioemocional; Autoestima; Clima escolar; Desarrollo profesional docente; Programas de desarrollo socioemocional.

The objective of this article is to describe a professional teacher's development program in socioemotional education and present some results of its impact in the self-esteem of students. It was executed in 12 subsidized private schools that attend economic vulnerable students from Preschool (4 years old) to High School (17 years old) and belong to an Educational Foundation in Santiago, Chile. It was executed the years 2007-2017, in training cycles of two years in each school. The most important topics that were addressed in the training given by experts, through theoretical sessions, workshops and educational materials, were defined with the schools. For example: Socioemotional learning in the school context; Variables that help to the construction of identity and positive self-esteem; Contribution of neurodiversity to socioemotional development; Resilience and socioemotional learning; School climate and social coexistence; Bullying and socioemotional development; Socioemotional learning and teacher well-being. The implementation of this program is described, emphasizing the value of socioemotional learning, how to develop competences in this area and how to insert it into the school context. Data are provided of 2,865 students evaluated pre-post intervention.

Keywords: Socioemotional education; Self-esteem; School climate; Teacher's professional development; Socioemotional development programs.

*Contacto: tita.marchant@gmail.com

issn: 1989-0397

www.rinace.net/riee/

https://revistas.uam.es/riee
Recibido: $\quad 12$ de noviembre de 2018

$1^{\text {a }}$ Evaluación: 24 de mayo de 2019

$2^{\text {a }}$ Evaluación: 20 de julio de 2019

Aceptado: $\quad 10$ de septiembre de 2019 


\section{Fundamentación Teórica}

Durante muchos años dominó la creencia en nuestra cultura de que existía un solo tipo de inteligencia, evaluada mediante el coeficiente intelectual, y que ella era determinante del éxito a alcanzar en la vida. Se priorizaba el desarrollo intelectual de los estudiantes por sobre el emocional y social. Se estimaba que estos últimos pertenecían al plano privado y por lo tanto cada familia debía preocuparse del desarrollo personal de sus hijos (Fernández-Berrocal y Ramos, 2002).

Investigadores, académicos y profesionales de la educación consideran al aprendizaje socioemocional un aspecto central a trabajar para favorecer el desarrollo integral de los alumnos (Garner, 2010; Haeussler y Milicic, 2014). La importancia del contexto escolar para el desarrollo socioemocional de niños y adolescentes resulta evidente al considerar la cantidad de horas y de años que pasan en dicho contexto y las relaciones y vínculos significativos que se establecen en éste. La socialización ocupa un rol privilegiado en la construcción de la narrativa que el niño hace de sí mismo y en el desarrollo de una autoestima positiva. Un modelo educativo centrado no solo en aspectos cognitivos y que integra otras perspectivas complementarias, posibilita que los alumnos lleguen a ser socialmente competentes, es decir que se lleven bien con otros, se comuniquen efectivamente, sean cooperativos, negocien con los demás para resolver sus problemas, sepan decir que no, sepan cuándo y dónde buscar ayuda y realicen una contribución positiva a sus familias y a la comunidad (Opengart, 2007). También favorece que, como futuros ciudadanos, reflexionen y pongan en práctica valores que facilitan la convivencia como el respeto, la participación y el diálogo (Bisquerra, 2003; Extremera y FernándezBerrocal, 2003). Un aspecto central de favorecer el aprendizaje socioemocional de los alumnos es el hacerlos tomar conciencia que todos somos parte de una comunidad, emergiendo como un tema importante, la valoración y aceptación de la diversidad (Alcalay, Berger, Milicic y Fantuzzi, 2012). En todas estas variables el profesor cumple un rol central y por lo tanto entregar herramientas y hacer actualizaciones de su formación parece fundamental y debiese ser parte de una política pública en educación.

Se ha planteado con bastante evidencia la importancia del desarrollo de las competencias emocionales de los docentes para enfrentar este desafío. Profesores y especialistas son agentes clave en la formación de vínculos, la convivencia, el clima social, la construcción de arquitectura cerebral, como figuras de apego secundario y tutores de resiliencia, ya que las relaciones positivas entre profesores y alumnos pueden incluso compensar en parte el déficit de apego con figuras de cuidado primario (Karreman y Vinherhoets, 2012). Los profesores además son significativos en el proceso de formación y desarrollo de la autoestima, ya que logran resultados con sus alumnos (Berger, Milicic, Alcalay y Torretti, 2014; Marchant, Milicic y Álamos, 2015). Sin embargo, los curriculum en la formación inicial docente, y los programas de actualización, normalmente ofrecen escasa formación teórica en aprendizaje socioemocional y en estrategias que lo favorezcan (Gaete, Gómez y Bascopé, 2016). Entre las necesidades percibidas de capacitación tanto por profesores, por expertos, como por las investigaciones, están los vínculos (vínculo profesor-alumno, vínculo profesor-padres, vínculo entre pares), el clima escolar, la disciplina, el cómo ayudar a los alumnos a desarrollar un proyecto de vida y los problemas crecientes en el área de la salud mental (Milicic y Arón, 2017). Tal como lo plantean Berger, Álamos y Milicic (2016), si los profesores no cuentan con posibilidades de capacitación en el área 
socioemocional les será más difícil desarrollarla en sus estudiantes y estarán más propensos al desgaste profesional con consecuencias para la salud mental de ambos.

Existe evidencia respecto a la importancia de un clima positivo para el desarrollo y bienestar de los estudiantes. El clima escolar corresponde al contexto social donde se desarrollan los estudiantes, comprendido como el patrón relacional propio de un curso o institución específica que implica los vínculos entre los miembros de ese grupo (Arón, Milicic, Sánchez y Subercaseaux, 2017). El clima escolar y el aprendizaje socioemocional de los estudiantes se relacionan de una manera circular y mutuamente reforzante. Un clima favorecedor de aprendizaje socioemocional se caracteriza por vínculos interpersonales que otorgan seguridad y contención a los alumnos, donde los adultos modelan y proveen instancias para practicar y aplicar habilidades sociales y afectivas y los estudiantes con mayores niveles de aprendizaje socioemocional contribuyen a la mantención del ambiente escolar positivo (Arón y Milicic, 2014; Cemalcilar, 2010). Berger, Milicic, Alcalay, Torretti, Arab y Justiniano (2009) señalan que en los ambientes escolares es necesario considerar variables como la relación de los estudiantes con sus profesores (u otros adultos en la escuela) y con sus pares. Las relaciones de amistad constituyen una fuente de apoyo al favorecer experiencias de participación, pertenencia y generar contextos de seguridad y de contención necesarios para que niños y adolescentes se desarrollen de forma positiva (Berger, Alcalay, Torretti y Milicic, 2011). Además de estas relaciones, es necesario considerar otras características del contexto escolar favorecedoras o limitantes del aprendizaje socioemocional: la estructura organizacional, los espacios físicos, la oferta programática curricular y extracurricular.

Los grandes avances de los últimos años en neurociencia han permitido profundizar en las bases biológicas del comportamiento socioemocional (Siegel, 2014). Investigaciones recientes, que sintetizan como se relaciona el mundo de la neurociencia y la educación han explorado las formas fundamentales en que interactúan las relaciones humanas y el cerebro para permitir el aprendizaje. Se concluye que éste es inherentemente social, que nuestras relaciones modelan el cómo funciona y se desarrolla y que un cerebro no receptivo inhibe el aprendizaje (Cozolino, 2013). También, que las experiencias tempranas tienen una influencia poderosa y duradera en su desarrollo y moldean la arquitectura cerebral (Céspedes, 2016) y que la atención genera acción cerebral, donde van las ideas, van las conexiones cerebrales (Siegel y Payne, 2018). Estos últimos autores plantean la importancia de focalizar la enseñanza en lo positivo y señalan que podemos ayudar a los niños a ser más creativos, abiertos y positivos, a ser más tolerantes, a controlar sus comportamientos y a desarrollar la capacidad de sobrellevar y resolver sus propios problemas cultivando su cerebro afirmativo, es decir, un cerebro interconectado cuyas partes trabajan juntas como un todo coordinado y equilibrado. Las relaciones entre padres e hijos, y también entre profesores y alumnos, afectan la receptividad del cerebro para incorporar nuevas experiencias y aprender de ellas. Hay que tomar conciencia que toda acción educativa crea cerebro y por lo tanto lo que no se hace, no crea cerebro o disminuye las posibilidades de desarrollo del cerebro social y del área socioemocional. En este proceso de formación, el rol del profesor como agente que construye arquitectura cerebral es central, especialmente para los alumnos que no recibieron tempranamente los nutrientes afectivos necesarios. Las relaciones positivas de los alumnos con sus profesores pueden compensar en parte el déficit de apego pobre con figuras de cuidado primario y son especialmente importantes para aquellos estudiantes que no han tenido oportunidades previas de desarrollar habilidades sociales y relaciones positivas y adaptativas (Karreman 
y Vinherhoets, 2012). Un contexto que no favorece relaciones interpersonales positivas y donde existen experiencias negativas como maltrato, crea un círculo vicioso que dificulta los procesos de regulación cognitiva, emocional y conductual. El contexto escolar debe entregar contención emocional, consuelo y apoyo cuando suceden eventos negativos, debe ser capaz de transformarse en un tutor de resiliencia, que capacite a los alumnos para enfrentar y superar situaciones adversas (Cyrulnik, 2009).

El aprendizaje socioemocional no es solo importante en la esfera afectiva, también tiene impacto para el rendimiento escolar y el bienestar general de niños y adolescentes en sus trayectorias de desarrollo. Hoy en día se sabe que el desarrollo socioemocional y el desarrollo cognitivo están relacionados. Una exhaustiva y actualizada revisión realizada por Izard (2009) confirma la relación indisoluble emoción-cognición y diversas investigaciones muestran que las intervenciones y programas de aprendizaje socioemocional no sólo mejoran la conducta de los estudiantes sino también incrementan su desempeño académico (Durlak, Weissberg, Dymnicki, Taylor y Schellinger, 2011). La generación de contextos en que se susciten respuestas emocionales positivas tenderá a producir en los alumnos un efecto de acercamiento hacia sus profesores, hacia sus pares y hacia el aprendizaje. Las emociones positivas facilitan la creatividad y están relacionadas con un mayor desarrollo cognitivo, toma de decisiones más eficientes, mayor tolerancia al fracaso y motivación intrínseca (Fernández-Abascal, 2009).

La autoestima, es un constructo central en el desarrollo socioemocional. Desde una mirada evolutiva, los cimientos que favorecen el desarrollo de una autoestima positiva se construyen durante la infancia, fundamentalmente a través de los mensajes que los adultos significativos transmiten a los niños acerca de sus competencias y de las experiencias de éxito que les logren ofrecer (Acevedo, 2008). Durante la preadolescencia y adolescencia existe asociación entre adecuados niveles de autoestima y relaciones interpersonales de calidad como factor protector (Bi, Ma, Yuan y Zhang, 2016). En estas etapas la baja autoestima se menciona con frecuencia como predictor de conductas de riesgo (Florenzano y Valdés, 2013). Sin embargo, esta tendencia varía, cuando se hacen adecuadas intervenciones en esta línea.

Actualmente existe una mayor conciencia respecto al rol que ocupa la autoestima en el bienestar emocional y la adaptación social. Haeussler y Milicic (2014) la señalan como clave para el éxito o fracaso personal. En ella se encuentra la base de la capacidad para responder de manera activa y positiva a las oportunidades que se presentan en los diferentes ámbitos de la vida, convirtiéndose así, en marco de referencia desde el cual las personas se proyectan y pieza fundamental de su desarrollo integral, equilibrio y bienestar emocional. Esta no solo es señalada como una característica básica de salud mental, sino también como factor protector que contribuye a una mejor salud a través de su función amortiguadora de experiencias negativas (Moksnes y Lazarewicz, 2016). La autoestima es un concepto significativo en educación pues se relaciona con aspectos relevantes como el rendimiento escolar, la motivación, el contacto del alumno consigo mismo y la conciencia de sí mismo, el desarrollo de un verdadero yo, la conexión con el propio cuerpo y el mundo interno, los vínculos, las relaciones interpersonales y la socialización, el desarrollo de nuestro cerebro y el aprendizaje, entre otros (Cozolino, 2013; Mayer y Salovey, 1997; Miller, 1991).

Las reformas educacionales en América Latina y el mundo han tenido la clara intención de incluir el desarrollo social y emocional de los estudiantes al formular los objetivos 
transversales. Sin embargo, éste aun no figura en los curriculum como un conjunto de objetivos a enseñar sistemáticamente. La transferencia y la instrumentalización de aquellos aspectos relacionados con el aprendizaje socioemocional implican un gran desafío que normalmente ha sido insuficientemente logrado en el sistema escolar.

El desafío que se plantea es cómo los profesores pueden dar seguridad a los estudiantes, como pueden crear climas en que los alumnos se sientan pertenecientes, apoyados y seguros y, complementariamente, como pueden favorecer en los estudiantes el desarrollo de conductas y actitudes que faciliten los vínculos y las relaciones positivas y nutritivas con sus pares, los profesores y el contexto en general. El sentimiento de pertenencia a una institución, la sensación de ser parte de una comunidad, el generar relaciones significativas en su interior, son todos elementos que apuntan a un apego escolar positivo. Un apego escolar seguro se relaciona con un contexto interpersonal en el cual existe respeto y valoración por el otro, pero además existen relaciones de intimidad, cercanía y pasarlo bien en conjunto. Los espacios lúdicos y el disfrute constituyen espacios de cercanía e intimidad que permiten generar modelos interpersonales positivos y nutritivos y sensación de pertenencia (Hamm y Faircloth, 2005). En la relación entre pares es fundamental que las instituciones educativas ayuden a desarrollar competencias socioemocionales como la empatía y autorregulación, así como también éticas, esenciales para la convivencia escolar y disciplina.

El Desarrollo Profesional Docente es un tema clave para que los individuos que trabajan en un sistema determinado integren mejoras y desarrollen capacidades. La principal característica de este concepto está dada por la posibilidad que los actores inviertan su energía en aprender nuevas destrezas y en generar prácticas más efectivas (Park, Petersen y Sun, 2013). Harris y Williams (2012) señalan que la construcción de capacidades debe ser sistémica, incluyendo a los diferentes actores y escenarios, para producir cambios en las prácticas profesionales y generar un círculo virtuoso, en donde cada ciclo da origen a uno mejor donde se vayan incorporando una o más mejoras a partir de evaluaciones constantes. De acuerdo a estos autores, el proceso de desarrollo de capacidades puede potenciarse desde afuera y desde adentro, siendo el elemento fundamental para que ello ocurra, la colaboración (Harris y Williams, 2012). Esta visión lleva a la necesidad de formar en aprendizaje socioemocional no solo a profesores aislados sino a toda la comunidad escolar.

El programa que se presenta en este artículo se centró en favorecer las competencias socioemocionales de los docentes como figuras claves en la formación de los alumnos. Este se desarrolló durante los años 2007-2017, en ciclos de dos años por colegio, e impactó a más de 1.000 profesionales que atienden a más de 14.000 alumnos pertenecientes a una Fundación Educacional. Se describe el diseño y la implementación de este programa que tuvo como modelo de base la teoría del aprendizaje socioemocional y la teoría sistémica. Su enfoque tanto de promoción como de prevención buscó desarrollar competencias socioemocionales en profesores y alumnos como un factor protector frente a los riesgos en la salud mental en atención a que fue realizado en colegios que atienden alumnos vulnerables económicamente. 


\section{Relato de la experiencia}

Al definir y diseñar una estrategia de intervención en aprendizaje socioemocional, en establecimientos educacionales, se hace necesario responder varias preguntas:

- ¿Cuáles son las necesidades de capacitación percibidas por el equipo directivo y los profesores? ¿Ellos perciben que requieren más capacitación? Si la respuesta es afirmativa ¿a quiénes se va a capacitar?, ¿en qué temas?, ¿con qué metodología?, ¿por cuánto tiempo?

- ¿Cómo se conocerá la realidad de la institución? ¿Se entrevistará a los directivos y profesores? ¿Se evaluará a los alumnos? ¿En qué cursos? ¿Con qué instrumentos?

- ¿Cómo se trabajará la coherencia Proyecto Educativo Institucional-Programa? ¿Cómo se intencionará la transferencia de lo aprendido al contexto escolar y al aula?

- ¿Cómo se apoyará a los alumnos que se definan como foco de intervención? ¿Cómo se hará seguimiento a las estrategias definidas para apoyar a estos alumnos?

- ¿Qué sistema de evaluación y de registro de información se utilizará para conocer la realidad y medir el impacto del programa en la comunidad educativa?

A continuación, se presenta la muestra, la respuesta o definición que se tomó frente a cada una de estas interrogantes y algunos resultados alcanzados.

\subsection{Muestra}

La muestra de profesionales capacitados fue de 1.153 directivos, profesores y especialistas, que, desde sus respectivos roles, atendían a los 14.073 alumnos de los 12 colegios de la institución. Esta se presenta en detalle en el cuadro 1.

Cuadro 1. Muestra de profesionales participantes

\begin{tabular}{lcccc}
\hline & $\mathbf{N}^{\circ}$ ProfeSORES & $\mathbf{N}^{\circ}$ DIRECTIVOS & $\mathbf{N}^{\circ}$ ESPECIALISTAS & $\mathbf{N}^{\circ}$ ALUMNOS \\
\hline Colegio 1 & 97 & 6 & 34 & 1.522 \\
Colegio 2 & 95 & 6 & 25 & 1.479 \\
Colegio 3 & 93 & 6 & 20 & 1.585 \\
Colegio 4 & 81 & 6 & 43 & 1.618 \\
Colegio 5 & 30 & 5 & 16 & 509 \\
Colegio 6 & 99 & 6 & 28 & 1.611 \\
Colegio 7 & 92 & 6 & 36 & 1.657 \\
Colegio 8 & 85 & 6 & 26 & 1.682 \\
Colegio 9 & 30 & 5 & 10 & 552 \\
Colegio 10 & 63 & 5 & 20 & 1.022 \\
Colegio 11 & 23 & 4 & 11 & 454 \\
Colegio 12 & 22 & 4 & 9 & 382 \\
\hline Total & 810 & 65 & 278 & 14.073 \\
\hline Fut
\end{tabular}

Fuente: Elaboración propia con datos de 2017.

El total de alumnos evaluados en autoestima fue de 2865. La autoestima de los alumnos se evaluó pre-post intervención de $3^{\circ}$ a $8^{\circ}$ básico. El detalle de la muestra se presenta en el cuadro 2. 
Cuadro 2. Muestra de alumnos evaluados pre-post intervención

\begin{tabular}{cc}
\hline \multirow{2}{*}{ CURSo } & TAE: TEST DE AUTOESTIMA DEL ESCOLAR \\
\cline { 2 - 2 } & $\mathbf{n}$ \\
\hline $3^{\circ}$ básico & 519 \\
$4^{\circ}$ básico & 461 \\
$5^{\circ}$ básico & 508 \\
$6^{\circ}$ básico & 482 \\
$7^{\circ}$ básico & 460 \\
$8^{\circ}$ básico & 435 \\
\hline Total & 2.865 \\
\hline
\end{tabular}

Fuente: Elaboración propia.

Adicionalmente se hizo un estudio de seguimiento de 240 alumnos de $3^{\circ}$ a $7^{\circ}$ básico que presentaron Baja o Muy Baja Autoestima y con los cuales se hizo un programa de intervención.

\subsection{Relato de la experiencia: Preguntas y respuestas}

$1^{a}$ pregunta: ¿Cuáles son las necesidades de capacitación percibidas por el equipo directivo y los profesores?

Los directivos y profesores de cada colegio coincidieron en que había temas de aprendizaje socioemocional que requerían profundizar: cómo asumir un rol activo en el desarrollo de una autoestima e identidad positiva de los estudiantes, cómo ayudar a los alumnos en la creación de vínculos y formulación de proyectos de vida, cómo crear climas que favorezcan la convivencia, y cómo fortalecer el propio desarrollo socioemocional.

Se optó por:

- Capacitar a toda la comunidad educativa de cada colegio: los profesores (PK a IV ${ }^{\circ}$ medio), los directivos superiores y especialistas.

- Hacer la capacitación con expertos en desarrollo socioemocional externos, apoyados por los profesionales de la propia institución.

- Hacerla durante períodos de 2 años, entregando tres jornadas de formación: una inicial de dos días, una segunda de dos días (6 meses después), una final de un día (9 meses después de la segunda).

- Capacitar a grupos de máximo 120 personas, es decir, 2 colegios grandes o 4 colegios pequeños, cada dos años.

- Capacitar con una metodología combinada entre conferencias generales, buscando un paradigma compartido, y talleres por ciclo, a grupos de máximo 30 personas, para hacerla experiencial. Se dieron tareas entre jornadas de capacitación a fin de facilitar la transferencia de lo aprendido al aula.

Los temas definidos como fundamentales del área, en cada ciclo de capacitación, fueron:

- Aprendizaje socioemocional en el contexto escolar.

- Paradigma de la inteligencia emocional en los alumnos.

- Apego escolar.

- Importancia del juego. 
- Variables que favorecen la construcción de identidad y autoestima positiva.

- Aporte de la neurodiversidad al desarrollo socioemocional.

○ Resiliencia y aprendizaje socioemocional.

- Clima escolar y convivencia social.

- Intimidación escolar y desarrollo socioemocional.

○ Relación y vínculo familia-escuela.

- Aprendizaje socioemocional y bienestar docente.

- Qué aporta la perspectiva del género a la educación.

- Procesos comunicacionales en el contexto escolar.

- Desafío de las nuevas tecnologías en el desarrollo cognitivo y emocional de los estudiantes.

- Propuestas de trabajo en aprendizaje socioemocional en el contexto escolar. $2^{a}$ pregunta: ¿Cómo se conocerá la realidad de la institución?

Se definió en primer lugar conocer las instancias preventivas de cada colegio para desarrollar el área socioemocional. Se hicieron grupos focales para recoger lo existente. Las estrategias se explicitaron en cada colegio, se compartieron entre colegios, y cada uno enriqueció las propias en base a lo aprendido de los expertos y de lo valorado en otros.

En segundo lugar, se definió evaluar la autoestima de los alumnos con instrumentos estandarizados, tipo screening o tamizaje, que permitiesen conocer la realidad e identificar a los alumnos que más preocupasen en el tema. Dado que en Chile solo existen instrumentos estandarizados para educación básica se optó por evaluar a los alumnos de $3^{\circ}$ a $8^{\circ}$ básico, vía auto-reporte, al iniciar y finalizar cada una de las intervenciones.

Adicionalmente, se implementó un sistema de reevaluación cada dos años, en todos los colegios ya capacitados, para mantener actualizadas las listas de alumnos a focalizar.

$3^{a}$ pregunta: ¿Cómo se trabajará la coherencia Proyecto Educativo-Programa? ¿Cómo se intencionará la transferencia de lo aprendido al contexto escolar y al aula?

Antes de iniciar el programa se conoció el Proyecto Educativo Institucional (PEI) de cada uno de los 12 colegios. Para intencionar la coherencia PEI-Programa, impactar a la comunidad educativa en sus lineamientos fundamentales y apoyar la transferencia se realizaron múltiples acciones:

- Se asignaron tareas entre jornadas de capacitación. Estas se relacionaron con evaluación de alumnos, transferencia de actividades vividas en la capacitación a la sala de clases y lectura de libros y documentos.

- Se definieron alumnos foco. Los alumnos foco se definieron como aquellos identificados por el Test TAE con Baja Autoestima (una desviación estándar bajo lo esperado) y con Muy Baja Autoestima (a más de una desviación estándar bajo lo esperado).

- Se enriqueció el Material de Desarrollo Socioemocional existente en las Bibliotecas Generales y de Aula incorporando libros de la línea psicoeducativa de alfabetización emocional y de juegos (Milicic y López de Lérida, 2011). Adicionalmente se formaron Bibliotecas-Profesor en cada establecimiento incorporando libros, talleres y artículos sobre aprendizaje socioemocional, además de las conferencias y talleres 
trabajados en ciclo de formación.

- Se acordó perfeccionar y ampliar el Programa de Orientación y Familia existente. En Chile éste tiene un espacio curricular asignado por el Ministerio de Educación de una hora semanal de $4^{\circ}$ básico a $\mathrm{II}^{\circ}$ medio. Al ampliarlo se quiso intervenir tempranamente en la educación emocional de los estudiantes para potenciar la mayor neuroplasticidad y también influir en las relaciones interpersonales y toma de decisiones marcadoras del proyecto de vida de los adolescentes. Tanto a los cursos básicos (Kínder a $3^{\circ}$ básico) como a los terminales (III ${ }^{\circ}$ y IV ${ }^{\circ}$ medio), se les asignó una hora curricular semanal para el programa. Para perfeccionarlo y ampliarlo fue fundamental:

- Conocer lo existente, escuchar a los actores del sistema y validar las experiencias positivas realizadas. Fue importante producir sinergia y no competencia entre programas.

- Estructurar el Programa de Orientación y Familia PK-IV medio en áreas y etapas a desarrollar con alumnos y apoderados. Se definieron cinco áreas (Autoconocimiento, Proyecto de Vida, Afectividad y Sexualidad, Relaciones Interpersonales e Inserción Social) y cinco etapas (Preescolar, $1^{\circ}-3^{\circ}$ básico, $4^{\circ}-6^{\circ}$ básico, $7^{\circ}$ básico- $I^{\circ}$ medio, $\mathrm{II}^{\circ}-\mathrm{IV}^{\mathrm{o}}$ medio). Para cada una se definieron características evolutivas, metodologías, objetivos y actividades.

- Hacerle seguimiento semestral al porcentaje de cumplimiento de su ejecución por profesor jefe.

- En cada colegio se programaron dos instancias formativas adicionales para profesores:

- Una hora mensual de capacitación de profesores jefes en el Programa Orientación y Familia.

- Un Consejo de Profesores al mes para profundizar en temas de Aprendizaje Socioemocional.

$4^{a}$ pregunta: ¿̨Cómo se apoyará a los alumnos definidos como foco de intervención?

Se diseñó una estrategia de intervención para apoyar a alumnos Baja Autoestima y Muy Baja Autoestima. Cada colegio se comprometió a implementar esta estrategia básica, mínima y común, en forma sistemática, y a enriquecerla de acuerdo a su realidad y posibilidades. El apoyo a los alumnos foco fue asumido principalmente por los profesores jefes como guías de los alumnos Baja Autoestima y por los encargados del Programa de Integración Escolar (PIE) como guías de los alumnos Muy Baja Autoestima. Los Encargados de Orientación y Familia de cada colegio realizaron seguimiento semestral a los responsables de los alumnos foco.

A continuación, se detallan las estrategias básicas definidas para los alumnos foco:

\section{Apoyo de Profesores Jefes a alumnos Baja Autoestima:}

- Dos entrevistas profesor-alumno por semestre.

- Seguimiento informal cotidiano y del trabajo académico de ellos.

- Seguimiento intencionado de alumnos en el Área Formación (hora semanal de Orientación y Familia y de Consejo de Curso). 
- Dos entrevistas semestrales profesor-apoderados de cada alumno.

- Revisión de indicadores generales (anotaciones positivas y negativas en el Libro de Clases, promedio de notas en los subsectores de Lenguaje-MatemáticaHistoria y Ciencias) para detectar posibles repitentes o desertores del sistema y tomar medidas remediales por establecimiento.

- Uso del material existente en Bibliotecas de educación socioemocional.

○ Premiación de aspectos destacados en estos alumnos.

- Definición del apoyo a estos alumnos como una tarea colegio, que compromete a directivos, profesores jefes y de subsectores de aprendizaje.

o Incorporación de estrategias y conocimientos aprendidos en el ciclo de capacitación.

2. Apoyo de un Encargado, normalmente el Encargado del Programa de Integración Escolar (PIE), a alumnos Muy Baja Autoestima:

- Recopilación de antecedentes de cada alumno (académicos, disciplina, asistencia alumno, asistencia apoderados a reuniones colegio, atención por especialista en el colegio o fuera de éste, participación en actividades extraprogramáticas, entre otros).

○ Dos entrevistas encargado-alumno por semestre.

- Dos entrevistas semestrales encargado-apoderados de cada alumno.

- Derivación a especialista cuando corresponda. Buen uso de las redes asistenciales.

- Seguimiento de derivaciones a especialistas.

- Uso del material existente en Bibliotecas de educación socioemocional.

- Premiación de aspectos destacados en estos alumnos.

○ Trabajo coordinado de encargado y profesores jefes.

- Incorporación de estrategias y conocimientos aprendidos en el ciclo de capacitación.

$5^{a}$ pregunta: ¿Qué sistema de evaluación y de registro de información se utilizará para conocer la realidad y medir el impacto del programa en la comunidad educativa?

Se acordó evaluar el programa a través de los resultados de autoestima de los estudiantes, asumiendo que una capacitación efectiva requiere una transferencia eficiente al aula. Se evaluaron los alumnos de tercero a octavo básico con un test estandarizado en Chile que la mide vía auto-reporte: el TAE-Alumno (Marchant, Haeussler y Torretti, 2002-2016) y que tiene adecuados índices de validez y confiabilidad (Alfa de Cronbach: 0,79; Coeficiente Kuder Richardson 20: 0,79; Validez concurrente con el Test Piers-Harris: ProductoMomento de Pearson: 0,88). Se conservaron los resultados de cada alumno pre-post intervención en dos de los puntajes clave que entrega el test seleccionado: Puntaje T y categoría de autoestima. Tras procesar esta información por curso, nivel de escolaridad, género, en cada colegio y en el total de colegios, se pudieron realizar:

- Estudios sobre la autoestima de alumnos de educación básica, de sectores de pobreza, por nivel de grado y género.

- Estudios de seguimiento (pre-post) de los alumnos foco. 


\subsection{Algunos resultados de la experiencia}

\subsubsection{Estudio por nivel de grado y género}

A continuación, se presentan los resultados de un estudio hecho con los datos de 2.865 alumnos, de $3^{\circ}$ a $8^{\circ}$ básico, de 5 colegios de la institución, evaluados con el test TAEAlumno (1.435 hombres y 1.430 mujeres) en noviembre 2014 .

En los Puntajes T se analizaron los promedios, las desviaciones estándar y el nivel de significación estadística de las diferencias de la muestra (Valor-p, Test Sidak, Test T) de acuerdo a nivel de escolaridad, a género y a nivel de escolaridad y género.

En los cuadros 3, 4 y 5 se presentan los resultados por nivel de escolaridad (3), por género (4) y por nivel de escolaridad y género (5), en Puntajes T.

Cuadro 3. Resultados TAE- Alumno por nivel de escolaridad, en Puntajes T

\begin{tabular}{lcccc}
\hline \multirow{2}{*}{ CURSO } & \multicolumn{3}{c}{ TAE PUNTAJE T 2014 } & \multirow{2}{*}{ SIDAK } \\
\cline { 2 - 4 } & $\mathbf{N}$ & $\mathbf{X}$ & $\mathbf{S T D}$ & $\mathrm{AB}$ \\
\hline $3^{\circ}$ básico & 519 & 51,2 & 12,7 & $\mathrm{~A}$ \\
$4^{\circ}$ básico & 461 & 52,2 & 13,1 & $\mathrm{AB}$ \\
$5^{\circ}$ básico & 508 & 50,5 & 13,4 & $\mathrm{AB}$ \\
$6^{\circ}$ básico & 482 & 50,5 & 13,8 & $\mathrm{AB}$ \\
$7^{\circ}$ básico & 460 & 50,2 & 12,7 & $\mathrm{~B}$ \\
$8^{\circ}$ básico & 435 & 48,8 & 12,1 & 0,006 \\
\hline Total & 2865 & 50,6 & 13 & \\
\hline
\end{tabular}

Fuente: Elaboración propia.

El resultado general da cuenta que en los alumnos de $3^{\circ}$ a $8^{\circ}$ básico aparecen diferencias estadísticamente significativas entre niveles de escolaridad observándose una baja de puntajes en la medida que los alumnos avanzan en ésta. Los Puntajes T más altos se alcanzan en $3^{\circ}-4^{\circ}$ básico y los más bajos en $8^{\circ}$ básico.

Cuadro 4. Resultados TAE-Alumno por género, en Puntajes T

\begin{tabular}{|c|c|c|c|c|c|}
\hline & & \multicolumn{3}{|c|}{ PUNTAJE T TAE. 2014} & \multirow{2}{*}{ Test T } \\
\hline & & $\mathbf{N}$ & $\mathrm{X}$ & STD & \\
\hline \multirow{2}{*}{ Género } & Hombre & 1435 & 51,8 & 12,7 & A \\
\hline & Mujer & 1430 & 49,4 & 13,2 & $\mathrm{~B}$ \\
\hline Total & & 2865 & 50,6 & 13 & $<0,001$ \\
\hline
\end{tabular}

Fuente: Elaboración propia.

En cuanto al análisis por género, se encuentran diferencias estadísticamente significativas presentando los hombres una autoestima más positiva que las mujeres.

Los resultados muestran que hubo diferencias estadísticamente significativas entre hombres y mujeres por nivel de escolaridad y género en la muestra total y en $7^{\circ}$ y $8^{\circ}$ básico. También se observó una mayor dispersión en los Puntajes T de las mujeres en relación a los hombres. En mujeres éstos oscilaron entre T46.3 y T51.9 y en hombres entre T50.9 y T53.2.

Cuadro 5. Resultados TAE-Alumno por nivel de escolaridad y género, en Puntajes T 


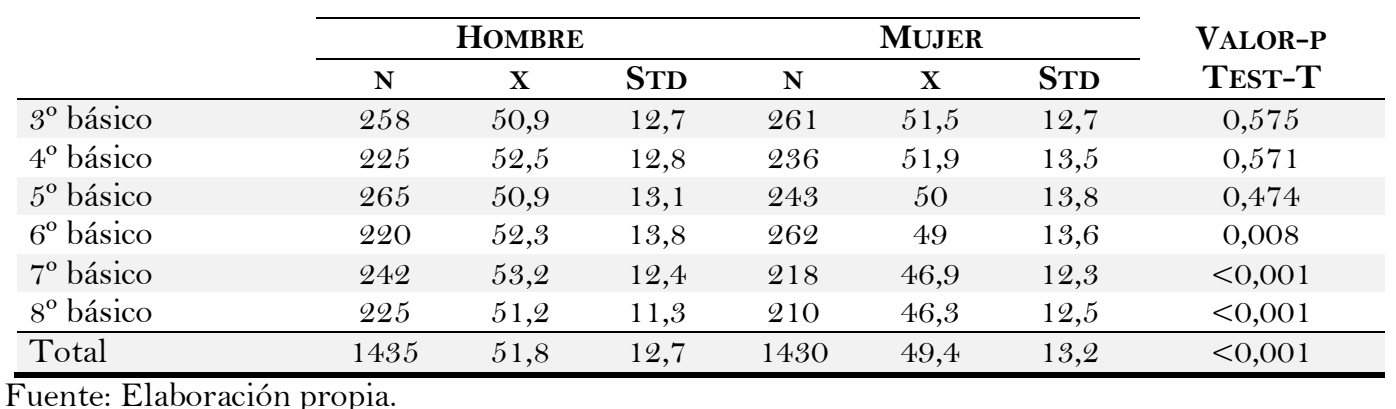

Fuente: Elaboración propia.

\subsubsection{Estudio de nivel de impacto}

A continuación, se presentan los resultados de un estudio de seguimiento de 240 alumnos con Baja Autoestima y Muy Baja Autoestima, de $3^{\circ}$ a $7^{\circ}$ básico, pertenecientes a 5 colegios, evaluados en dos períodos de tiempo. Estos alumnos fueron evaluados con el test TAEAlumno los años 2012 y 2014. Se hizo seguimiento a los Puntajes T (Valor-p, Test Chi Cuadrado de Pearson) y a la permanencia o cambio de categoría de autoestima (\%).

Los cuadros 6 y 7 muestran el seguimiento de los estudiantes que presentaron Baja Autoestima y Muy Baja Autoestima en Puntajes T.

Cuadro 6. Seguimiento 188 alumnos Baja Autoestima TAE-Alumno, 2012-2014, en Puntajes T

\begin{tabular}{cccccccc}
\hline & \multicolumn{3}{c}{ PUNTAJE T 2012 } & \multicolumn{3}{c}{ PUNTAJE T 2014 } & VAlOR-P \\
\cline { 2 - 6 } & $\mathbf{n}$ & $\mathbf{x}$ & $\mathbf{S t d}$ & $\mathbf{n}$ & $\mathbf{x}$ & $\mathbf{S t d}$ & TEST-T \\
\hline Total $3^{\circ} \mathrm{a} 7^{\circ}$ & 188 & 35,9 & 2,8 & 188 & 41,7 & 11,2 & $<0,001$ \\
\hline
\end{tabular}

Fuente: Elaboración propia.

Cuadro 7. Seguimiento 52 alumnos Muy Baja Autoestima TAE-Alumno, 2012-2014, en Puntajes T

\begin{tabular}{cccccccc}
\hline & \multicolumn{3}{c}{ Puntaje T 2012 } & \multicolumn{3}{c}{ Puntaje T 2014 } & Valor-p \\
\cline { 2 - 6 } & $\mathbf{n}$ & $\mathbf{x}$ & Std & $\mathbf{n}$ & $\mathbf{x}$ & Std & Test-T \\
\hline Total $3^{\circ}$ a $7^{\circ}$ & 52 & 25,4 & 2,9 & 52 & 38,6 & 11,2 & $<0,001$ \\
\hline
\end{tabular}

Fuente: Elaboración propia.

El análisis pre-post mostró diferencias significativas tanto en alumnos Baja Autoestima como Muy Baja Autoestima $(\mathrm{p}<0,001)$. Los alumnos con Baja Autoestima mejoraron sus Puntaje T de T 35,9 a T 41,7 y los con Muy Baja Autoestima mejoraron sus Puntajes T de $\mathrm{T} 25,4$ a $\mathrm{T} 38,6$.

El cuadro 8 presenta el seguimiento de los alumnos Baja Autoestima y Muy Baja Autoestima $(n=240)$ por categoría de Autoestima. En ésta se analizó el porcentaje de alumnos que permanecieron en la misma categoría o que cambiaron de categoría ya sea pasando a una categoría más alta o más baja.

Entre los resultados obtenidos por categoría de autoestima cabe destacar que:

- $\quad 53 \%$ de los alumnos $3^{\circ}$ a $7^{\circ}$ básico 2012, con Baja Autoestima (n=188), la mejoraron alcanzando una Autoestima Normal. Un 32\% permaneció en Baja Autoestima y un 15,4\% disminuyó su Autoestima y obtuvo Muy Baja Autoestima.

- $\quad 73 \%$ de los alumnos $3^{\circ}$ a $7^{\circ}$ básico 2012 con Muy Baja Autoestima (n=52), mejoraron su autoestima (33\% alcanzó una Autoestima Normal y 40\% cambió a Baja 
Autoestima). Un 26,9\% permaneció en Muy Baja Autoestima.

Cuadro 8. Seguimiento alumnos Baja Autoestima y Muy Baja Autoestima TAE-Alumno, 2012-2014. Categoría de Autoestima

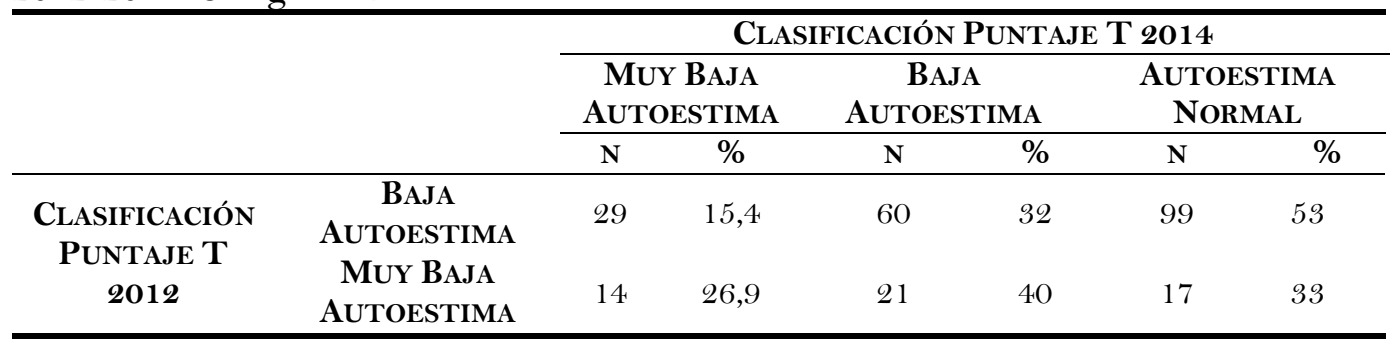

Fuente: Elaboración propia.

\section{Discusión y conclusiones}

El trabajo colaborativo, interactivos sistemático, con vínculo profundo con los profesores, nos lleva a algunas conclusiones:

En primer lugar, resultó evidente que los profesores tienen un alto interés en el tema autoestima y aprendizaje socioemocional y se comprometen con él. El estudio aporta evidencia en favor de que una debida capacitación a los profesores influiría positivamente en el desarrollo de autoestima de sus alumnos y plantea que las intervenciones que incluyen a profesores y directivos constituirían un camino estratégico para promover el desarrollo socioemocional de niños y jóvenes. Estas conclusiones son consistentes con las de trabajos internacionales en el tema (Denham, Bassett y Zinsser, 2012; Garner, 2010; Vostanis, Humphrey, Fitzgerald, Deighton y Wolpert, 2013). Para lograr este compromiso parece fundamental crear espacios que permitan conocer lo que los profesores hacen para favorecer los vínculos profesor-alumno, entre profesores y con la comunidad. Conocer permite validar, reconocer lo que se hace, transferir las experiencias exitosas e incorporar nueva información.

En segundo lugar, surgió de la experiencia que para lograr esto, parece fundamental que los directivos y profesores de cada establecimiento piensen y reflexionen juntos y estén atentos a las oportunidades que están ofreciendo a niños y jóvenes para promover u obstruir el aprendizaje socioemocional, a hacer intervenciones en los períodos que resulten más críticos en la construcción de la identidad y se pregunten qué tipo de alumno quieren entregar y están entregando a la sociedad. La capacitación de todos podría ayudar a darle coherencia al proyecto educativo y crea comunidad (Jellison, Jabbar, Germain y Dinning, 2018).

En tercer lugar, desde la capacitación, surgieron conclusiones relevantes:

- Hay temas que preocupan a todas las comunidades educativas y que parecen ser los que son necesarios de profundizar en las capacitaciones y que se listaron anteriormente. Estos surgieron desde la consulta a las comunidades en distintos períodos de tiempo y desde la experiencia de los expertos.

- Fue muy significativo tomar los temas atingentes del desarrollo emocional de los alumnos vía charlas magistrales dictadas por expertos y vía talleres vivenciales, en que directivos y profesores podían compartir sus vivencias y experiencia con éstos en el ámbito escolar. Al vivir los profesores los talleres por un lado se facilita la transferencia al aula, se les entrega un modelo que permite vivenciar el cómo se 
pueden implementar los conceptos en actividades a realizar en la sala de clases. Por otro lado, al recoger estas experiencias el programa gana en amplitud y no solo impacta en el desarrollo socioemocional de los alumnos sino de los adultos a cargo de la educación de éstos y su quehacer profesional (Marchant et al., 2015). Ellos mejoran en desempeño académico, autoestima personal y profesional, cambian sus atribuciones de éxito y fracaso de los alumnos haciéndose más responsables de su aprendizaje y perfeccionan su rol de ser formadores integrales y no solo guías de aprendizaje (Torretti, Lucchini, Marchant, Prats y Recart, 2007).

- Fue muy significativo para la comunidad educativa el capacitarlos a todos juntos, el hacerlos partícipes de un programa que impactaba a los alumnos desde Pre-Kínder a $\mathrm{IV}^{\circ}$ medio y en que todos eran responsables de su éxito desde el lugar en que se desempeñaban.

- Fue de gran valor el enfrentar a los docentes a una exposición prolongada en el tema que permitió retomarlo y profundizarlo una y otra vez, recogiendo experiencias vivenciales. Esto fue muy importante para lograr un proyecto global, coherente y para su transferencia al aula. A partir de este punto, se plantea la importancia de promover la formación continua de los profesores que les permita dedicar tiempo a actualizar conocimientos y competencias docentes.

- Fue necesario ampliar el foco de la capacitación. Originalmente esta se centraba en autoestima. En el transcurso del programa se ampliaron los objetivos y actividades a educación socioemocional.

En cuarto lugar, esta experiencia destaca la importancia de valorar y explicitar el trabajo preventivo que se hace en el área socioemocional en los colegios y muy especialmente en el Programa de Orientación y Familia. Este programa tiene espacio curricular y debiese tener objetivos claros, áreas y etapas definidas, y actividades a desarrollar adecuadas para cada ciclo de vida. También que es fundamental realizarle acompañamiento y seguimiento sistemático para asegurar su adecuada implementación. La capacitación por expertos mostró la necesidad y el valor que tendría el ampliar la cobertura del Programa de Orientación y Familia a fin de intervenir tempranamente en la educación emocional de los estudiantes y también en las relaciones interpersonales y en el desarrollo de proyectos de vida con los alumnos de educación media. Esto resultó efectivo ya que el compromiso y grado de influencia de los profesores y especialistas de los cursos iniciales y terminales incrementó fuertemente.

Esta experiencia también señala la importancia de la evaluación de los alumnos para conocer la realidad, poder identificar alumnos que requieren especial atención y medir el impacto de las intervenciones. En esta experiencia los datos se generaron al interior de una red de colegios que evalúa permanentemente el desempeño de los alumnos en los ámbitos académicos y no académicos. Se analizaron en detalle a fin de conocer la realidad e identificar a los estudiantes con mayores necesidades en cuanto al desarrollo de una autoestima positiva. Al evaluar a los alumnos pre-post (al iniciar las intervenciones y dos años después) se constató que los resultados generales mejoraban significativamente (Puntajes T) y que los alumnos Baja Autoestima y Muy Baja Autoestima disminuían significativamente. Los resultados del seguimiento hecho a los alumnos que presentaron Baja Autoestima, y con quienes se intencionó un trabajo en autoestima con sus profesores jefes, mostraron que los profesores pueden ser buenos agentes de aprendizaje 
socioemocional para sus estudiantes, pudiendo convertirse en formadores integrales de niños y adolescentes. La estrategia de "visualizar" a estos alumnos y establecer un vínculo más cercano con ellos y sus familias, reportó un alza significativa de autoestima. También que los alumnos Muy Baja Autoestima pueden ser ayudados en el contexto escolar con la colaboración de especialistas de los colegios y utilizando las redes de apoyo. La aplicación de planes remediales al interior de los establecimientos educacionales, con los profesores y especialistas, parece una medida adecuada, que refuerza la importancia de la toma de decisiones con base en evidencia. La sistematización de la información de autoestima también permitió realizar investigación, aportar evidencia científica al trabajo en el área y hacer difusión. Sería interesante hacer, a futuro, estudios de impacto y de seguimiento con grupo control a fin de verificar las conclusiones que se extraen de este estudio. Es importante mencionar el valor de iniciativas sistematizadas en Programas de Desarrollo Socioemocional, que ofrecen un contexto de oportunidades intencionadas para que los estudiantes desarrollen una autoestima positiva, orientando la labor docente en esta línea (Berger et al., 2014).

Finalmente, los estudios permitieron a la institución conocer la realidad en profundidad, poder intervenirla y evidenciar la efectividad del programa de capacitación. Es así como, por ejemplo, se constató que la institución hacía un buen trabajo preventivo en aprendizaje socioemocional en sus colegios. Los alumnos a pesar de provenir de sectores desaventajados económicamente, en las evaluaciones iniciales presentaban, en un alto porcentaje, un nivel de Autoestima Normal. Los resultados por nivel de escolaridad y género reflejaron que la autoestima es un concepto dinámico y que existirían edades o etapas que son más críticas para su desarrollo. Al constatar que la autoestima se deteriora después de $6^{\circ}$ básico, y especialmente en las mujeres, surgió la necesidad de fortalecer las intervenciones en esos grupos. Al indagar el como se estaba enfrentando el tema género en cada establecimiento y conocer las acciones que se realizaban tanto para hombres como mujeres en sala de clases, recreos y actividades extraprogramáticas se vio la necesidad de crear acciones y talleres solo orientados a mujeres. Los datos científicos evidenciaron la efectividad de este programa de aprendizaje socioemocional y permitieron destacar, el importante rol que cumplen directivos, profesores y especialistas en su desarrollo en el contexto escolar (Marchant, Milicic y Álamos, 2015; Marchant, Milicic y Pino, 2017).

Como equipo investigador, nos surgen algunas conclusiones que serían importantes de investigar con directivos y profesores:

En primer lugar, que es fundamental fortalecer los vínculos. En contextos vulnerables el desarrollo de vínculos profesor-alumno es especialmente relevante ya que los profesores pueden hacer la diferencia como figuras de apego secundario y tutores de resiliencia.

En segundo lugar, que la calidad, la equidad y la búsqueda de igualdad también pasa por el tema de desarrollo personal. Es importante que los establecimientos educacionales tengan un enfoque psicoeducativo que permita a los alumnos desarrollar lo socioemocional, crear vínculos, desarrollar un proyecto de vida. Los colegios debiesen impulsar que las personas salgan adelante, dar más instancias para el desarrollo personal, saber reconocer los talentos y desarrollarlos, y dar oportunidades a todos, especialmente a los que más los necesitan.

En tercer lugar, esta experiencia revela la importancia de construir una educación basada en las fortalezas más que en los déficits de los estudiantes, creando entornos educativos que otorguen oportunidades para el desarrollo de los talentos y donde las diferencias de 
género no se constituyan en discriminación. La presente estrategia de intervención en desarrollo socioemocional, en 12 colegios, que consideró la capacitación de docentes, directivos y especialistas, durante períodos de dos años, con expertos en desarrollo socioemocional externos, con evaluación de los alumnos para poder conocer la realidad y determinar los alumnos a focalizar, con desarrollo de estrategias de intervención y con seguimiento de éstas, con mejora del trabajo preventivo que se realiza y con desarrollo de investigación a fin de conocer la realidad en profundidad y el impacto de las intervenciones, demuestra que el trabajo riguroso en el área socioemocional rinde claros frutos y que los establecimientos educacionales son espacios privilegiados para su desarrollo.

\section{Referencias}

Acevedo, A. (2008). La buena crianza. Pautas y reflexiones sobre cómo criar con responsabilidad y alegría. Bogotá: Editorial Norma.

Alcalay, L., Berger, C., Milicic, N. y Fantuzzi, X. (2012). Aprendizaje socioemocional y apego escolar: favoreciendo la educación en diversidad. En I. Mena, M. R. Lissi, L. Alcalay y N. Milicic (Eds.), Diversidad y educación: miradas desde la psicología educacional (pp. 47-68). Santiago de Chile: Ediciones Universidad Católica.

Aron, A. M., Milicic, N., Sánchez, M. y Subercaseaux, J. (2017). Construyendo juntos: Claves para la convivencia escolar. Santiago de Chile: Agencia de Calidad de la Educación.

Berger, C., Alamos, P. y Milicic, N. (2016). El rol de los docentes en el aprendizaje socioemocional. En J. Manzo y M. R. García (Eds.), La transformación de las prácticas docentes (pp. 645-666). Santiago de Chile: Ediciones Universidad Católica.

Berger, C., Milicic, N., Alcalay, L. y Torretti, A. (2014). Learning and wellbeing program for third and fourth grade students: Impact description and evaluation. Revista Latinoamericana de Psicología, 46(3), 169-177.

Berger, C., Alcalay, L., Torretti, A. y Milicic, N. (2011). Socio-emotional well-being and academic achievement: Evidence from a multilevel approach. Psicología: Reflexão e Crítica, 24(2), 1-8.

Berger, C., Milicic, N., Alcalay, L., Torreti, A., Arab, M. P. y Justiniano, B. (2009). Socio-emotional Wellbeing in Educational Contexts: Perception of Chilean Students. Estudios sobre Educación, 17, 21-43.

Bi, Y., Ma, L., Yuan, F. y Zhang, B. (2016). Self-esteem, perceived stress, and gender during adolescence: interactive links to different types of interpersonal relationships. Journal of Psychology: Interdisciplinary and Applied, 150(1), 36-57. https://doi.org/10.1080/00223980.2014.996512

Bisquerra, R. (2003). Educación emocional y competencias básicas para la vida. Revista de Investigación Educativa, 21, 7-43.

Cemalcilar, Z. (2010). Schools as socialization contexts: Understanding the impact of school climate factors on students' sense of school belonging. Applied Psychology: An International Review, 59, 243-272. https://doi.org/10.1111/j.1464-0597.2009.00389.x

Céspedes, A. (2016). Tu cerebro. Un libro para adolescentes y para los que dejaron de serlo. Santiago de Chile: Ediciones B.

Cozolino, L. (2013). The Social Neuroscience of Education. Optimizing Attachment and Learning. Londres: W.W. Norton and Company. 
Cyrulnik, B. (2009). Autobiografía de un espantapájaros. Testimonios de resiliencia: el retorno a la vida. Barcelona: Gedisa.

Denham, S. A., Bassett, H. H. y Zinsser, K. (2012). Early childhood teachers as socializers of young children's emotional competence. Early Childhood Education Journal, 40, 137-143.

Durlak, J., Weissberg, R., Dymnicki, A., Taylor, R. y Schellinger, K. (2011). The Impact of Enhancing Students' Social and Emotional Learning: A Meta-Analysis of School-Based Universal Interventions. Child Development, 82(1), 405-432. https://doi.org/10.1111/j.1467-8624.2010.01564.x

Extremera, N. y Fernández-Berrocal, P. (2003). La inteligencia emocional: Métodos de evaluación en el aula. Revista Iberoamericana de Educación, 30, 1-12.

Fernández-Abascal, E. (2009). Emociones positivas. Madrid: Ediciones Pirámide.

Fernández-Berrocal, P. y Ramos, N. (2002). Corazones inteligentes. Barcelona: Kairos.

Florenzano, R. y Valdés, M. (2013). El adolescente y sus conductas de riesgo. Santiago de Chile: Ediciones UC.

Gaete, A., Gómez, V. y Bascopé, M. (2016). Temas de la agenda pública: ¿Qué le piden los profesores a la formación inicial docente en Chile? Santiago de Chile: Centro de Políticas Públicas.

Garner, P. (2010). Emotional competence and its influences on teaching and learning. Educational Psychology Review, 22(3), 297-321. https://doi.org/10.1007/s 10648-010-9129-4

Hamm, J. y Faircloth, B. (2005). The role of friendship in adolescents' sense of school belonging. New Directions for Child and Adolescent Development, 107, 61-78. https://doi.org/10.1002/cd.121

Harris, D. y Williams, J. (2012). The association of classroom interactions, year group and social class. British Educational Research Journal, 38(3), 373-397.

https://doi.org/10.1080/01411926.2010.548547

Haeussler, I. M. y Milicic, N. (2014). Confiar en uno mismo. Programa de desarrollo de la autoestima. Santiago de Chile: Catalonia.

Hoffman, D. (2009). Reflecting on social emotional learning: a critical perspective on trends in the United States. Review of Educational Research, 79(2), 533-556. https://doi.org/10.3102/0034654308325184

Ibarrola, B. (2017). Aprendizaje emocionante. Neurociencia para el aula. Barcelona: Ediciones SM.

Izard, C. E. (2009). Emotion theory and research: highlights, unanswered questions and emerging issues. Annual Review of Psychology, 60, 1-25. https://doi.org/10.1146/annurev.psych.60.110707.163539

Jellison, J., Jabbar H., Germain, E. y Dinning, J. (2018). Rethinking teacher turnover: longitudinal measures of instability in schools. Educational Researcher, 47(1), 62-75. https://doi.org/10.3102/0013189X17735813

Karreman, A. y Vinherhoets, A. (2012). Attachment and well-being: the mediating role of emotion regulation and resilience. Personality and Individual Differences, 53(7), 821-826. https://doi.org/10.1016/j.paid.2012.06.014

Marchant, T., Haeussler, I. M. y Torretti, A. (2016). TAE: Test de Autoestima-Escolar Evaluación del alumno vía auto-reporte y vía profesor. Santiago de Chile: Ediciones UC.

Marchant, T., Milicic, N. y Pino M. J. (2017). La autoestima en alumnos de $3^{\circ}$ a $8^{\circ}$ básico. Una mirada por nivel de escolaridad y género. Revista Iberoamericana de Evaluación Educativa, 1O(2), 111-117. https://doi.org/10.15366/riee2017.10.2.006 
Marchant, T., Milicic, N. y Álamos, P. (2015). Competencias socioemocionales: capacitación de directivos y docentes y su impacto en la autoestima de alumnos de $3^{\circ}$ a $7^{\circ}$ básico. Revista Iberoamericana de Evaluación Educativa, 8(2), 213-218.

Mayer, J. D. y Salovey, P. (1997). What is Emotional Intelligence? En P. Salovey y D. J. Sluyter (Eds.), Emotional development and emotional intelligence: Educational implications (pp. 3-31). Nueva York, NY: Basic Books.

Milicic, N. y Arón, A. M. (2017). Clima social escolar: ¿¿Un espacio tóxico o un espacio nutritivo?. Ciudad de México: Somos Maestras.

Milicic, N. y López de Lérida, S. (2011). Hijos con autoestima positiva. Santiago de Chile: Norma Ediciones.

Milicic, N., Alcalay, L., Berger, C. y Torretti, A. (2014). Aprendizaje socioemocional. Programa BASE (Bienestar y Aprendizaje Socioemocional) como estrategia de desarrollo en el contexto escolar. Santiago de Chile: Ariel.

Miller, A. (1991). El drama del niño dotado. Barcelona: Tusquets.

Moksnes, U. K. y Lazarewicz, M. (2016). The association between self-esteem and sense of coherence in adolescents aged 13-18 years-The role of sex and age differences. Personality and Individual Differences, 90, 150-154. https://doi.org/10.1016/j.paid.2015.10.049

Opengart, R. (2007). Emotional intelligence in the K-12 curriculum and its relationship to American workplace needs: a literature review. Human Resource Development Review, 6(46), 442-458. https://doi.org/10.1177/1534484307307556

Park, N., Peterson, C. y Sun, J. (2013). Positive psychology: research and practice. Terapia Psicológica, 31(1), 11-19. https://doi.org/10.4067/S0718-48082013000100002

Siegel, D. (2014). Tormenta cerebral. El poder y el propósito del cerebro adolescente. Madrid: Alba.

Siegel, D y Payne, T. (2018). El cerebro afirmativo del niño. Ayuda a tu hijo a ser más resiliente, autónomo y creativo. Barcelona: Penguin Random House.

Torretti, A., Lucchini, G., Marchant, T., Prats, A. y Recart, I. (2007). Informe final: caminar juntos. Programa de perfeccionamiento docente entre pares. Licantén 2001-2005. Santiago de Chile: Edición de Fundación Educacional Arauco.

Vostanis, P., Humphrey, N., Fitzgerald, N., Deighton, J. y Wolpert, M. (2013). How do schools promote emotional wellbeing among their pupils? Findings from a national scoping survey of mental health provision in English schools. Child and Adolescent Mental Health, 18, 151157. https://doi.org/10.1111/j.1475-3588.2012.00677.x

\section{Breve Cv de las autoras}

\section{Teresita Marchant Orrego}

Psicóloga de la Pontificia Universidad Católica de Chile y Magíster en Dirección y Gestión Escolar de Calidad Universidad del Desarrollo-Fundación Chile. Se ha desempeñado en cargos de dirección en el Departamento de Psicopedagogía del Liceo Alemán de Santiago (1979-1989), en Fundación Educacional Arauco (1990-2005) y en Fundación Belén Educa (2006-2015). Impartió docencia en el Programa de Magíster en Educación Especial de la Pontificia Universidad Católica de Chile (1980-1993). Actualmente es investigadora y asesora externa de Estudios y Consultorías Focus Ltda. (2015-2018) y asesora de Fundación Belén Educa (2016-2018). Es autora de varios artículos sobre trastornos de aprendizaje, lectura y desarrollo socio-emocional y coautora 
de libros como: Test de Desarrollo Psicomotor TEPSI; Pruebas de Dominio Lector Fundación Educacional Arauco; y TAE: Test de Autoestima del Escolar. ORCID ID: https://orcid.org/0000-0003-3883-3940. Email: tita.marchant@gmail.com

\section{Neva Milicic Müller}

Psicóloga, Magíster en Educación de la Pontificia Universidad Católica de Chile y profesora emérita de la misma Universidad. PhD de la Universidad de Gales, Gran Bretaña. Entre sus numerosas publicaciones se cuentan Madurez Escolar, Clínica Escolar, Educación y Diversidad y Aprendizaje Socioemocional, entre otros; y diversos artículos en revistas científicas internacionales como Mindfulness and Parenting: A Correlational Study of Non-meditating Mothers of Preschool Children (2016), Academic performance and personal and contextual dimensions of socioemotional learning: Evidence of its association in Chilean students (2014), Learning and wellbeing program for third and fourth grade students: Impact description and evaluation (2014). Ha sido conferencista invitada en diversos países de América Latina y en España. ORCID ID: https://orcid.org/0000-0002-4950-1901. Email: neva.milicic@gmail.com

\section{Paulina Soto Vásquez}

Psicóloga, Magister en Psicología Educacional de la Pontificia Universidad Católica de Chile. Se ha desempeñado como Docente de la Escuela de Educación de la Universidad San Sebastián y de la Escuela de Psicología de UNIACC. Actualmente, es directora ejecutiva del proyecto EducaAfecto subsidiado por Corfo, el cual favorece la formación integral de los estudiantes en establecimientos educacional del país, articulando Familia y Escuela. Es coautora del libro "7 principles to achive educational success". ORCID ID: https://orcid.org/000-0002-7820-1086.Email: pau.asv@gmail.com 